Int. J. Electrochem. Sci., 15 (2020) 5540 - 5551

\title{
Efficient Solar Cells Constructed with Lead Iodide Perovskite Templated by a 3-aminopropyl trimethoxysilane and methyltrimethoxysilane Mixed Monolayer
}

\author{
Qinghua Liu ${ }^{1,2, *}$, Dianlu Jiang ${ }^{3, * *}$, Qing Kang ${ }^{4}$, Feimeng Zhou ${ }^{3, * * *}$ \\ ${ }^{1}$ College of Chemistry and Chemical Engineering, Central South University, Changsha, Hunan \\ 410083, P.R. China \\ ${ }^{2}$ School of Chemistry and Chemical Engineering, Heze University, Heze 274015, China \\ ${ }^{3}$ Department of Chemistry and Biochemistry, California State University, Los Angeles, Los Angeles, \\ California 90032 \\ ${ }^{4}$ Institute of Surface Analysis and Chemical Biology, University of Jinan, Jinan, Shandong 250022, P. \\ R. China \\ *E-mail: liuqinghua-126@126.com; \\ **E-mail: djiang4@calstatela.edu \\ ***E-mail: $\underline{\text { fzhou@ calstatela.edu }}$
}

doi: $10.20964 / 2020.06 .51$

Received: 24 January 2020 / Accepted: 16 March 2020 / Published: 10 May 2020

\begin{abstract}
A lead iodide perovskite solar cell has been designed using a mixed monolayer of (3aminopropyl)trimethoxysilane (APS) and methyltrimethoxysilane (MTMS) self-assembled on a compact $\mathrm{TiO}_{2}$ layer to template the perovskite film growth. The dispersed aminopropyl groups at the surface, upon conversion to $\left(\mathrm{CH}_{2}\right)_{3} \mathrm{NH}_{3}{ }^{+} \mathrm{I}^{-}$, form the bottommost layer of the perovskite film. The effect of the APS/MTMS compositions in the mixed monolayers on the performance of the perovskite solar cells (PSCs) was investigated by measuring the power conversion efficiency. We developed a surface modification procedure with which the [APS]/[MTMS] ratios in solution match exactly the compositions of APS and MTMS in the resultant mixed monolayers. At the [APS]/[MTMS] ratio of 50/50, the distance between two adjacent $\mathrm{Si}$ atoms matches well with the lattice constant of the lead iodide perovskite crystal, and the power conversion efficiency of PSCs increases from 13.2\% (without the monolayer) to $17.5 \%$. X-ray diffraction patterns suggest that the templated perovskite growth is along the (110) plane, with enhanced orientation and crystallinity. The increase in electron lifetime of the solar cell indicates that the number of photohole/electron recombination sites is reduced and charge transport at the perovskite film $/ \mathrm{TiO}_{2}$ interface is facilitated.
\end{abstract}

Keywords: Mixed organosilane monolayer, Covalent anchor, Orientation, template, Lead iodide perovskite 


\section{FULL TEXT}

(C) 2020 The Authors. Published by ESG (www.electrochemsci.org). This article is an open access article distributed under the terms and conditions of the Creative Commons Attribution license (http://creativecommons.org/licenses/by/4.0/). 